\title{
Automated classification and quantification of F-actin-containing ruffles in confocal micrographs
}

\author{
Qing Yi and Marc G. Coppolino \\ University of Guelph, Guelph, ON, Canada \\ BioTechniques 40:745-756 (June 2006) \\ doi 10.2144/000112162
}

\begin{abstract}
Membrane ruffles are actin-rich protrusions of the plasma membrane that can be observed on the surface of many cell types. Phase contrast and fluorescent microscopy are widely used in the analysis of ruffles, which are commonly identified in cells stained with fluorescently labeled phalloidin. Currently, comparison of cellular ruffle formation under different experimental conditions is generally qualitative or semiquantitative. Ruffle structures are often defined using manual tracing and thresholding methods. Here, we report the rapid and accurate segmentation of ruffles from two-dimensional confocal projections of cells using an automated method based on well-established image processing and analysis methods. Lineshaped ruffles were detected using line detectors and were then separated from the filtered images. Automated categorizing of the segmented line structures enabled accurate quantification of the ruffles. This automated approach is efficient and reliable and hence can serve as a powerful tool in studies of the mechanism of ruffle formation.
\end{abstract}

\section{INTRODUCTION}

Membrane ruffles are thin cell surface membrane protrusions that are enriched with filamentous actin (F-actin). These structures have been widely observed in cultured eukaryotic cells, including both stationary and migrating fibroblasts (1-3). F-actin-rich membrane ruffles have also been observed in vivo, for example in embryonic cells (4). Formation of ruffles in migrating cells is known to be closely related to establishing cell polarity; in general, the formation of ruffles is restricted to the leading edge of migrating cells (5). The polarized distributions of cellular components, such as matrix-metalloproteinase 2 (MMP2) (6), aquaporin 1 (AQP-1) (7), high-affinity $\alpha_{\mathrm{v}} \beta_{3}$ integrin (8), phosphatidylinositol-4, 5-bisphosphate $\left[\mathrm{PI}(4,5) \mathrm{P}_{2}\right](9)$, and endocytosed epidermal growth factor receptors (EGFRs) (10) have also been observed to correlate with sites of ruffle formation. While recent studies indicate that ruffles are compartments of inhibited actin turnover that correlate with inefficient lamellipodia adhesion (11), the biochemical mechanisms that regulate ruffle formation are not completely understood.
In analyses of ruffles, phase contrast and fluorescent microscopy are widely used techniques. Ruffles are commonly identified through their unique morphology, observed in cells stained with fluorescently labeled phalloidin to reveal F-actin-containing structures. In dorsal views of two-dimensional (2-D) cell images, the morphology of F-actin-rich ruffles is quite consistent between different cell lines, including Chinese hamster ovary-K1 (CHO-K1), COS-7, NIH 3T3, and embryonic fibroblasts, and can be described as sharp sinuous line-shaped structures (1). Currently, comparison of cellular ruffle formation under different experimental conditions is generally qualitative or semiquantitative. Ruffle structures are often defined using manual tracing and thresholding methods (12-14). These manual operations are time-consuming and thus limit the number of images that can be analyzed. The repeatability of these manual operations can be poor and may also reduce the accuracy of the analyses.

Here, we report that rapid and accurate segmentation of ruffles from 2-D images of cells can be achieved through an automated method based on well-established image processing and analysis methods. Line-shaped ruffles were detected using line detectors, and the ruffles were accurately separated from surrounding cellular structures through thresholding methods. Automated categorizing of the segmented line structures enabled accurate quantification of the ruffles. This automated approach is efficient and reliable and hence can serve as a powerful tool in studies of the mechanism of ruffle formation.

\section{MATERIALS AND METHODS}

\section{Cells, Transfection, and Reagents}

CHO-K1 cells were maintained in Dulbecco's modified Eagle's medium (DMEM; Sigma-Aldrich, St. Louis, MO, USA) containing $10 \%$ fetal bovine serum (FBS; Sigma-Aldrich), at $37^{\circ} \mathrm{C}$ and $5 \% \mathrm{CO}_{2}$. Phorbol-12-myristate13-acetate (PMA) was purchased from Sigma-Aldrich. pEGFP-N1 vector was purchased from Clontech Laboratories (Palo Alto, CA, USA). Rhodaminephalloidin and 4'6-diamidino-2-phenylindole (DAPI) were purchased from Molecular Probes (Eugene, OR, USA), and fluorescent mounting medium was obtained from DakoCytomation (Carpinteria, CA, USA).

\section{Cell Stimulation and Immunofluorescent Labeling}

To induce ruffle formation, cells were washed with cold phosphatebuffered saline (PBS) three times and detached with $5 \mathrm{mM}$ EDTA in PBS. Detached cells were then plated subconfluently on $10 \mu \mathrm{g} / \mathrm{mL}$ fibronectin-coated glass coverslips and allowed to attach and spread on the coverslips for $3 \mathrm{~h}$ in serum-free DMEM at $37^{\circ} \mathrm{C}$ with $5 \%$ $\mathrm{CO}_{2}$. Plated cells were treated with 500 nM PMA in DMEM or DMEM alone for $10 \mathrm{~min}$, to induce ruffles or serve as control, respectively. Cells were fixed with $4 \%$ paraformaldehyde at $4^{\circ} \mathrm{C}$ for $30 \mathrm{~min}$ and permeabilized with $0.1 \%$ Triton ${ }^{\circledR} \mathrm{X}-100$ in PBS for 10 min. Fixed and permeabilized cells were washed three times with PBS and incubated in 5\% skim milk/PBS blocking solution for $1 \mathrm{~h}$. After three washes in PBS, cells were incubated with $0.04 \mathrm{U} / \mathrm{mL}$ 
rhodamine-phalloidin for $30 \mathrm{~min}$. Cells were washed three times and then stained with $300 \mathrm{nM}$ DAPI for $5 \mathrm{~min}$ to label nuclei. After two more washes, cells were mounted on glass slides using fluorescent mounting medium.

\section{Image Acquisition and Outlining Cell Boundaries}

Labeled cells were viewed using a Leica TCS SP2 confocal imaging station equipped with a Leica DMIRE2 inverted microscope and a Leica $63 \times$ oil immersion lens (Leica Microsystems, Heidelberg, Germany). Cells were optically sectioned along the z-axis from ventral to dorsal surfaces. The voxel size was $0.23 \mu \mathrm{m}$ in each of the $\mathrm{x}, \mathrm{y}$, and $\mathrm{z}$ dimensions. From the acquired $z$-series, a projection image, which offered a high-resolution 2-D overview, was generated for each cell. The projection image was generated by overlaying the optical sections on top of each other (the ZProject/Sum Slices function in ImageJ software).

Cell boundaries were detected in cells stained with rhodamine- phalloidin and confirmed in phase contrast images and in samples transfected with green fluorescent protein (GFP) as a marker of the cytoplasm. To define cell boundaries in projected images, we used Otsu's method, which separates image components based on analyzing the variance of their intensity values (15). After being processed by Otsu's method, a projected cell image, in which the cellular components were separated from the background, was generated for each cell. On this projection image, the boundary for a cell was easily defined by the outer most nonzero pixels around the cell image. The boundary was demarcated in the $\mathrm{x}$ and $\mathrm{y}$ dimensions, which was acceptable given that there were generally few deep invaginations along cell boundaries.

\section{Image Processing}

ImageJ for Windows (NIH Images), was used to isolate ruffle structures from within the outlined cell boundaries in the projection images. For isolating ruffles, projection images were generated from the dorsal portion of cells, defined as sections above the fourth $\mathrm{z}$-section from the ventral surface, which did not contain prominent $\mathrm{F}$-actin stress fibers. Isolation of ruffles was achieved through three steps: first, the line-shaped structures on the 2-D projection image were located using a line detection method2-D difference-of-Gaussian (DoG) filtering. The 2-D DoG filtering was implemented according to Equation 1:

$$
\begin{gathered}
\operatorname{DoG}(x, y)=\frac{1}{2 \pi \sigma_{1}^{2}} \exp \left(-\frac{x^{2}+y^{2}}{2 \sigma_{1}^{2}}\right) \\
-\frac{1}{2 \pi \sigma_{2}^{2}} \exp \left(-\frac{x^{2}+y^{2}}{2 \sigma_{2}^{2}}\right)
\end{gathered}
$$

[Eq. 1]

in which the image generated with the 2-D Gaussian filter of $\sigma_{2}$ (1.28) was subtracted from the image generated with that of $\sigma_{1}(0.8)$. Here, the ratio $1: 1.6$ of $\sigma_{1}: \sigma_{2}$ (Equation 1) was used to minimize the bandwidth and maintain sensitivity (16). Second, the detected lines on the 2-D DoG filtered image were further segregated by a threshold

\begin{tabular}{|c|c|c|}
\hline Moment Descriptors & $\begin{array}{l}\text { Equation } \\
\text { (Reference) }\end{array}$ & Description \\
\hline$M 1, M 2$ & Given by (23) & $\begin{array}{l}\text { These two lowest order moment invariants are independent of object position, } \\
\text { size and orientation, and are useful for object recognition. }\end{array}$ \\
\hline Measured Metrics & - & Description \\
\hline $\operatorname{Area}(A)$ & & $\begin{array}{l}\text { The area of an image feature is expressed as the total pixel numbers in the } \\
\text { feature. }\end{array}$ \\
\hline Maximum Feret diameter $\left(F_{\max }\right)$ & & $\begin{array}{l}\text { The maximum Feret diameter is the longest distance between any two points on } \\
\text { the boundary of the image. }\end{array}$ \\
\hline Major, Minor & & $\begin{array}{l}\text { The major axis (Major) and minor axis (Minor) are the primary and secondary } \\
\text { axis of the best fitting ellipse to the image feature. }\end{array}$ \\
\hline Bounding Rectangle & & $\begin{array}{l}\text { The bounding rectangle is defined as the smallest rectangle enclosing the } \\
\text { selection. }\end{array}$ \\
\hline Anisometry & $\frac{\text { Major }}{\text { Minor }}$ (25) & $\begin{array}{l}\text { Anisometry has values of } 1.0 \text { (a perfect circle) and higher. Filamentous structures } \\
\text { often have large Anisometry values. }\end{array}$ \\
\hline Curl & $\frac{F_{\max }}{\left(\frac{P}{2}-\frac{2 A}{P}\right)}$ & $\begin{array}{l}\text { The denominator in Curl is used to estimate Fiber Length (17). For fibers that are } \\
\text { nearly straight, this approaches } 1.0 \text {, and it becomes much smaller as the fiber is } \\
\text { more curled. }\end{array}$ \\
\hline
\end{tabular}

Table 1. Shape Parameters Used in this Protocol 
method-the fixed percentage setting (17). The threshold value for each individual 2-D DoG filtered image was adjusted to include $15 \%$ of the projected cell area within the positive extreme value on the 2-D DoG filtered images. Several fixed percentage settings were tried, including 10\% and $20 \%$; however, a setting of $15 \%$ reliably produced images containing structures that were consistent with the ruffles observed in the cells by visual inspection. Third, among all the segmented line structures, line features with the longer dimension of the bounding rectangle (Table 1) greater than 20 pixels (approximately $4.6 \mu \mathrm{m}$ ) were considered as potential ruffles. The 20 pixel lower limit was determined by trial and error. Values below this resulted in images containing a large population of structures that precluded manual classification and therefore could not be comparatively analyzed. The images of extracted ruffle structures were then used for further classification.

\section{Image Classification and Ruffle Quantification}

Image classification was used to categorize the isolated potential ruffle images. Automated image classification was achieved through logistic regression model. Logistic regression (18) is a well-established statistical method for computational classification associated with binary outcomes (e.g., ruffles or non-ruffles). A set of training image features was visually inspected and categorized as either ruffles or nonruffles manually. Shape parameters, including three geometric descriptors, Form Factor, Anisometry, and Curl, and two moment invariants, $M I$ and $M 2$, were collected for these training images (Table 1). The distribution of each shape parameter was analyzed for significance of difference between ruffles and non-ruffles. Multivariate logistic regression analysis was then used to assess the relationship between image group membership and the five shape parameters. A logistic regression equation was then built for automated image classification using the output of the multivariate logistic regression analysis.
An integrated macro (see the supplementary material available at www. BioTechniques.com) for ImageJ was developed to achieve rapid implementation of the ruffle quantification protocol. Ruffle quantification was done by calculating a ruffle index for each cell. After image classification, the total area of ruffle features of a cell was summed for that cell. A ruffle index was calculated for each cell through dividing the summed ruffle area in a cell by the projected area of that cell and multiplying by a factor of 100 .

\section{Statistical Analysis}

Statistical analyses were done using Microsoft $^{\circledR}$ Excel $^{\circledR} 2002$ and SPSS ${ }^{\circledR}$ 12.0 (SPSS Inc., Chicago, IL, USA). Analysis for significance of difference between two groups was carried out either by Student's $t$-test when normal distribution can be assumed or by Mann-Whitney U test for very skewed data sets. Logistic regression, using SPSS 12.0 , was used to analyze the relationship between image group membership (ruffles or non-ruffles) and the five shape parameters. For logistic regression, the dependent variable was the group membership of image objects. The independent variables or predictors were M1, M2, Anisometry, Form Factor, and $\mathrm{Curl}$. The square root transformation for M2 (sM2), and Box-Tidwell transformation for $\mathrm{Curl}[\mathrm{Curl} \times \operatorname{Ln}(\mathrm{Curl})]$ were used to achieve best fit (18).

\section{RESULTS AND DISCUSSION}

\section{Extraction of Ruffle Images}

In cultured $\mathrm{CHO}-\mathrm{K} 1$ cells, F-actincontaining ruffles generally protrude into the space above the cell. When ruffling $\mathrm{CHO}-\mathrm{K} 1$ cells were imaged with a laserscanning confocal microscope, it was clear that the ruffles mainly appeared on the dorsal portion of the cell, with ruffles at different heights above the surfaces of cells. Dorsal optical sections in a $\mathrm{z}$-series were therefore combined to generate a projection image for each cell. The resulting image was a sum of all dorsal F-actin structures, including sinuous line-shaped ruffles (Figure 1A). We chose to acquire cell images by optical sectioning using confocal microscopy to obtain images of high resolution while developing our method of analysis. Images acquired using other methods, such as conventional light microscopy, would also be suitable for this protocol; although, in some cases, preprocessing, such as deconvolution, may be necessary to achieve sufficient resolution.

In one-dimensional (1-D) intensity profiles of ruffle structures, rapid intensity changes over short distances are clearly observed (Figure 1, A and G). This type of intensity change is characteristic of line structures (19). Line structures can be detected by a genre of image processing methods called edge detectors. The 2-D DoG filter is an edge detector with easy implementation and good performance in line detection $(20,21)$. In CHO-K1 cells, we found that ruffles are thin lines with their width generally limited to 2-3 pixels (and no greater than 5 pixels) or approximately $1.2 \mu \mathrm{m}$. Based on this observation, a $\sigma_{1}$ value of 0.8 , which is equivalent to the $3 \times$ 3 2-D Gaussian filter, was found to be satisfactory during 2-D DoG filtering (Figure 1B). The negative intensity values generated by the $2-\mathrm{D}$ DoG filter were stored in the format of 32-bit floating-point grayscale images, and hence no rescaling was necessary.

On the 2-D DoG filtered images, line structures had high and positive intensity values, and the regions surrounding those line structures had low and negative values. The remaining regions had intermediate intensity values fluctuating around zero (Figure 1, B and G). This distribution was consistent for the 2-D DoG filtered cell images and was reflected by their Gaussian-shape intensity histograms (Figure 1F). There are three parts in the intensity histograms: (i) the positive values (the right end), (ii) the negative values (the left end), and (iii) the medium values (the middle part), which contained the detected lines, regions surrounding lines, and the remaining regions, respectively. This pattern provides a simple method to segment the detected lines from other structures. A minimal threshold intensity value can be assigned to each individual 
A
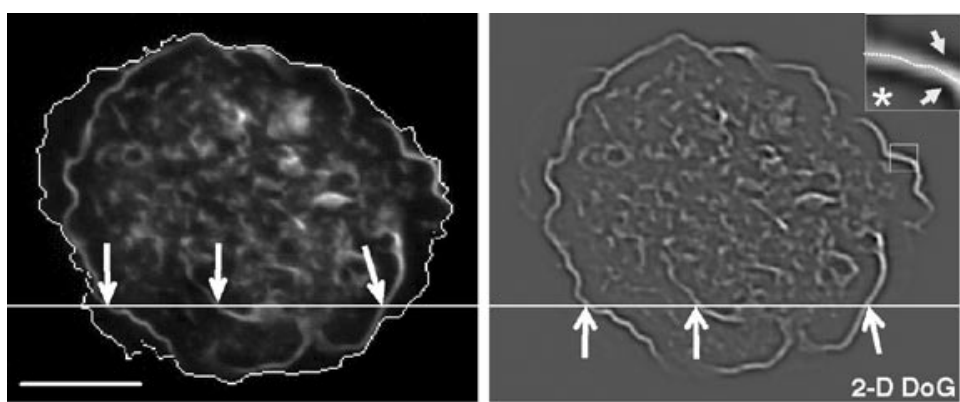

C
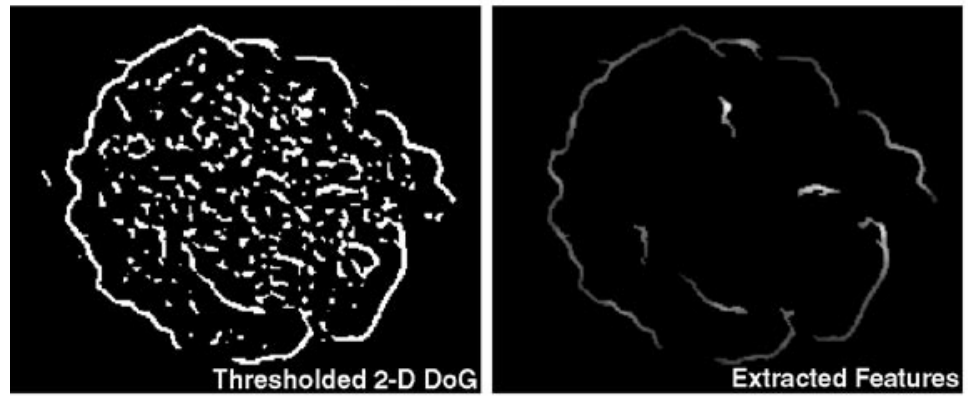

E
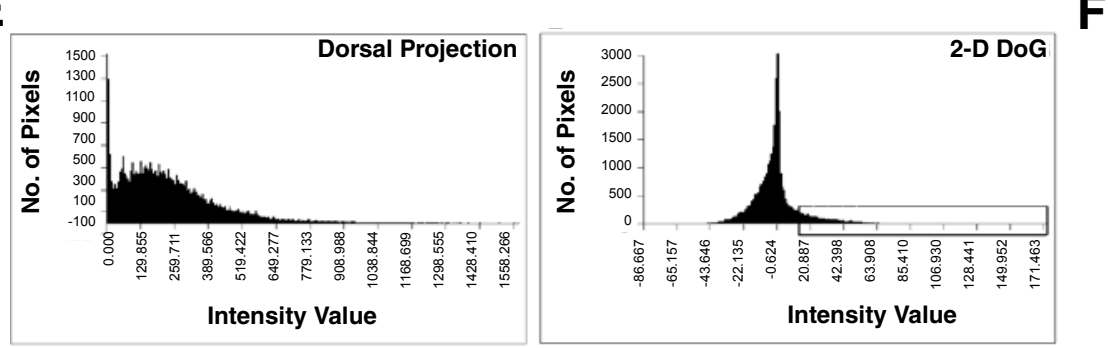

G

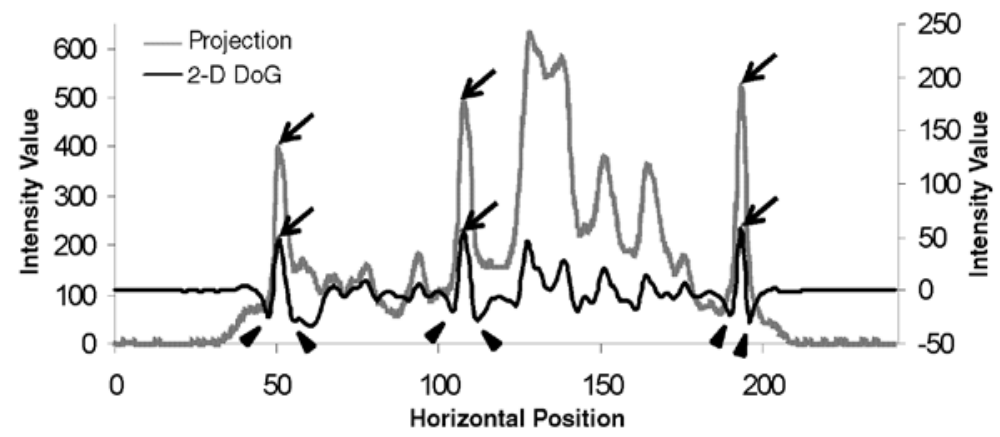

2-D DoG filtered image, such that, on the 2-D DoG filtered image, cellular regions with their intensity values no smaller than the threshold values were retained and other regions were cleared. This threshold method-fixed percentage setting (17)—was used to find the minimal threshold value for each 2-D DoG filtered image. We used the recommended $15 \%$ fixed percentage (17). The total area of the retained cellular regions accounted
B

Figure 1. Extraction of ruffle features from confocal cell images. (A) A projection image of F-actin-containing structures in a phorbol-12myristate-13-acetate (PMA)-stimulated Chinese hamster ovary $(\mathrm{CHO})$ cell stained with rhodamine-labeled phalloidin was generated for a $\mathrm{CHO}$ cell labeled with phalloidin. The cell was optically sectioned by confocal microscopy, and the dorsal portion of the $\mathrm{z}$-series was used to generate the projection image. Outline of cell boundary is indicated. (B) The dorsal projection image in panel A was processed with the two-dimensional (2-D) difference-of-Gaussian (DoG) filter. The 2D DoG filtering was implemented by subtracting the image filtered with 2-D Gaussian of kernel 1.28 from 2-D Gaussian of kernel 0.8. As shown on the insert, which is the enlarged image of the boxed region, the line feature (dashed curve) has positive and high intensity values (lighter color), the regions surrounding the line (arrows) have low and negative values (darker color), and the remaining regions (asterisk) have intermediate intensity values (gray color). (C) The 2-D DoG filtered image in panel B) was thresholded using the fixed percentage setting. The total area of these features accounts for $15 \%$ of the calculated projection cell area. (D) Image features with the longer dimension of the bounding rectangle greater than 20 pixels were extracted from the dorsal projection images in panel C. (E and F) Intensity histograms for the dorsal projection image in panel A and 2-D DoG filtered image in panel B, respectively. The Gaussian shaped histogram in panel $\mathrm{F}$ was consistent for all 2-D DoG images. Boxed region in panel $\mathrm{F}$ contains cellular features above the minimum threshold value (14.74 for this image). (G) Two one-dimensional (1-D) intensity profiles represent every pixel on the lines in panels A and B, respectively. The horizontal position ( $\mathrm{x}$-axis) starts from the leftmost pixels on the images. The intensity curves for the lines in panels A and B are plotted using the left and right $\mathrm{y}$-axes, respectively. Three ruffles (arrows in panels A and B) are also indicated by corresponding arrows on the profiles. The curves show that ruffles represent lines on this intensity profile. The black curve shows that ruffles (arrows) have high and positive values, regions surrounding ruffles (arrowheads) have low and negative values, and the remaining regions are intermediate in value. Scale bar in panel A represents $10 \mu \mathrm{m}$.

potential ruffle images from $945 \mathrm{CHO}$ cells in 38 experiments, was manually classified. Ruffles were defined as sinuous, line-shaped structures with sharp boundaries. The majority of the extracted image features were ruffles, with the percentage of ruffle images being $73.9 \%$ and that of nonruffles being $26.1 \%$. Since manual classification was based on inspecting the detailed shape characteristics of extracted image features, we sought to 
A

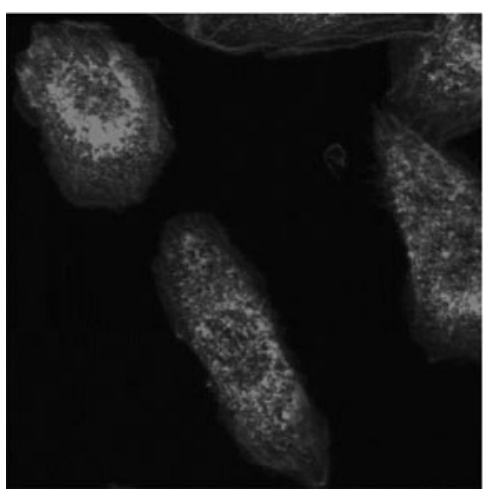

B

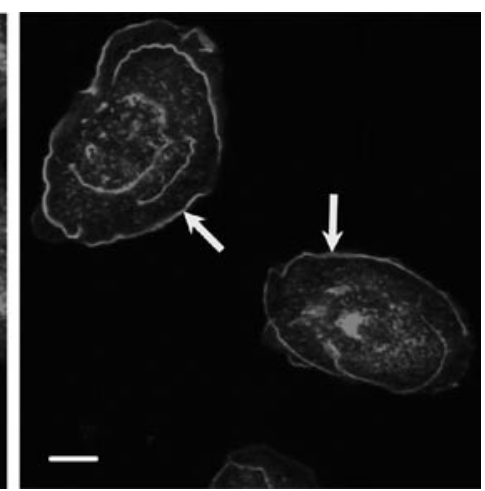

C

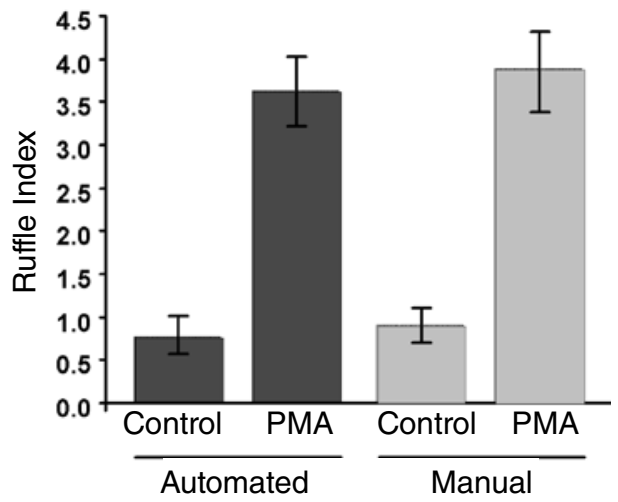

Figure 2. Automated quantification of phorbol-12-myristate-13-acetate (PMA)-induced ruffle formation. Chinese hamster ovary (CHO) cells were treated with 500 nM PMA in Dulbecco's modified Eagle's medium (DMEM) or DMEM alone for 10 min, fixed, stained with rhodamine-phalloidin, and imaged as described. (A) Control, untreated cells. (B) PMA-treated cells. Arrows point to ruffle structures. (C) Calculated ruffle indices (expressed as mean \pm SEM) from manual and automated classification. Control, cells were treated with DMEM alone; PMA, cells were treated with $500 \mathrm{nM}$ PMA for 10 min. Student's $t$-test showed that the differences between the control and PMA-treated samples are significant for both methods of analysis, with two-tailed $P$ values being 0.00312 $(<0.01)$ for the automated protocol and $0.00251(<0.01)$ for the manual scoring. Results are from three independent experiments with $>25$ cells per experiment. Scale bar represents $10 \mu \mathrm{m}$.

automatically classify features through computational analysis of the shape parameters in the extracted images. Five shape parameters, Form Factor, Anisometry, Curl, M1, and M2, (Table 2) were measured for all the training images. Values of each parameter, expressed as mean \pm standard error, tended to be larger for ruffles than for non-ruffles (Table 2). Statistical analysis (Mann-Whitney U test) verified that the distribution of each parameter was significantly different between ruffles and non-ruffles (Table 2). This result indicates the existence of correlation between values of each shape parameter and image group membership. Multivariate logistic regression analysis was further used to assess the reliability of the parameterbased classification. All five shape parameters, Form Factor, Anisometry, Curl, $M 1$, and $M 2$, were entered as independent variables (or predictors). Transformation was made for two of the shape parameters for best fit, as determined by trial and error; square root value of $M 2$ was used, and Curl was Box-Tidwell transformed. The group membership of image features was entered as dependent variables with 1 assigned to ruffles and 0 assigned to non-ruffles. The results showed that the logistic regression model, and coefficients for each shape parameter and the constant (or intercept), were all significant (Table 2). The statistical significance was also verified by $50-50$ crossexamination. This further indicates a correlation between shape parameters and image group membership and suggests the ability to classify image features through their shape parameters. A logistic regression equation (Equation 2) was then built from the results of the logistic regression analysis of training images

$$
\begin{aligned}
& \ln \left[\frac{P}{1-P}\right]=-3.097+21.154 \times M 1-13.146 \times \\
& s M 2-0.336 \times F+0.361 \times A+15.216 \times b t C
\end{aligned}
$$

[Eq. 2]

\begin{tabular}{|c|c|c|c|c|c|c|c|c|}
\hline \multirow[t]{2}{*}{ Parameters } & \multicolumn{2}{|c|}{$\begin{array}{l}\text { Values of Parameters } \\
(\text { mean } \pm \text { SEM })\end{array}$} & \multirow[t]{2}{*}{$\begin{array}{c}\sigma^{\mathrm{a}} \\
\text { (2-tailed) }\end{array}$} & \multirow[t]{2}{*}{$\beta^{\mathbf{b}}$} & \multirow[t]{2}{*}{$\begin{array}{l}\text { SEM } \\
\text { for } \beta\end{array}$} & \multirow[t]{2}{*}{$\begin{array}{c}\sigma \\
\text { for } \beta\end{array}$} & \multicolumn{2}{|c|}{$\begin{array}{c}95 \% \mathrm{Cl} \\
\text { for } \exp (\beta)\end{array}$} \\
\hline & $\begin{array}{c}\text { Ruffles } \\
\text { (4371 images) }\end{array}$ & $\begin{array}{l}\text { Non-Ruffles } \\
\text { (1546 images) }\end{array}$ & & & & & Lower & Upper \\
\hline M1 & $1.370 \pm 0.0129$ & $0.597 \pm 0.00427$ & 0.000 & 21.154 & 1.273 & 0.000 & $1.269 \times 10^{8}$ & $1.864 \times 10^{10}$ \\
\hline$M 2^{c}$ & $2.151 \pm 0.0483$ & $0.235 \pm 0.00575$ & 0.000 & -13.146 & 1.267 & 0.000 & $2.340 \times 10^{-5}$ & $1.630 \times 10^{-7}$ \\
\hline Anisometry & $6.449 \pm 0.0482$ & $2.838 \pm 0.0268$ & 0.000 & 0.361 & 0.103 & 0.000 & 1.171 & 1.756 \\
\hline Curld & $0.858 \pm 0.0018$ & $0.638 \pm 0.00323$ & 0.000 & 15.216 & 1.349 & 0.000 & $2.886 \times 10^{5}$ & $5.709 \times 10^{7}$ \\
\hline Constant & - & - & - & -3.097 & 0.503 & 0.000 & - & - \\
\hline \multicolumn{9}{|c|}{$\begin{array}{l}\mathrm{Cl} \text {, confidence interval. } \\
\text { aThe distributions of each measured parameter were evaluated for significance of difference. } \\
\text { bThe relationship between image group memberships and measured parameters was evaluated by multivariate logistic regression. } \\
\text { cThe value of } M 2 \text { was square root transformed in the multivariate logistic regression. } \\
\text { dThe value of } C \text { Curl was Box-Tidwell transformed in the multivariate logistic regression. }\end{array}$} \\
\hline
\end{tabular}

Table 2. Associations Between Image Group Memberships and Measured Parameters 
in which $P$ represents the probability for an image feature to be a ruffle, $s M 2$ is the square root transformed $M 2, F$ is Form Factor, $A$ is Anisometry, and btC is the Box-Tidwell transformed Curl. This equation was further used for automated image classification.

\section{Evaluation of Ruffle Quantification Protocol}

CHO cells were used to evaluate the application of this ruffle quantification protocol. Dramatic ruffles formed in PMA-treated cells, whereas few ruffles were visible in nontreated cells (Figure 2, A and B). An integrated macro for ImageJ was used for rapid implementation of the ruffle quantification protocol. In this evaluation, the extracted ruffle images were classified both manually and automatically. The automated classification showed that ruffles accounted for $76.3 \%$ and nonruffles accounted for $23.7 \%$ of segregated structures. This distribution is very close to that obtained by manual classification. The automated classification was carried out using Equation 2 , and the probability cut-off value was set as 0.5. A structure was classified as ruffle when the probability was greater than 0.5 and was classified as nonruffle otherwise. Comparison of results obtained by the automated method with those obtained by the manual method showed that, overall, $92.8 \%$ of image structures were consistently classified as either ruffles or non-ruffles between the two methods. When expressed as mean \pm standard error, the ruffle indices calculated from automated classifications $(0.761 \pm 0.205$ and $3.62 \pm 0.4$ for non-induced and PMA-induced cells, respectively) were very close to those from manual inspections $(0.812 \pm$ 0.189 and $3.81 \pm 0.402$ for non-induced and PMA-induced cells, respectively) (Figure 2C). The difference in ruffle indices between non-induced and PMA-induced cells was statistically significant for both methods of classification. Hence, the quantitative results using automated classification reliably reflected the visible difference in ruffle formation between PMA-induced and non-induced cells.

Automated classification and quantification of platelet-derived growth factor (PDGF)-induced ruffling in NIH $3 \mathrm{~T} 3$ cells was also conducted using Equation 2 and the parameter values in Table 2. This yielded satisfactory results (see Supplementary Figure S1), but underestimated the level of ruffle formation. This resulted from differences in the morphology of ruffles in the two cell types and indicates that to apply Equation 2 to other cell types, which may have unique ruffle features, regression coefficients need to be generated from data sets obtained using that cell type.

We quantified ruffles in images acquired form dorsal sections of cells. This eliminated many F-actincontaining structures, such as stress fibers and lamellipodia, which are found in the ventral sections of these cells. While it possible that non-ruffle structures will be detected using our method, the classification protocol, using parameters in Table 2, places restrictions on object shape that make this unlikely. We have confirmed this by analyzing F-actin-containing ruffles together with plasma membrane structures (using GFP-tagged membrane markers or anti-integrin antibodies, data not shown). Using our method, we were unable to detect ruffle-like structures that did not correspond with plasma membrane structures.

In summary, we report a method for the automated classification and quantification of F-actin-containing ruffles from fluorescence micrographs of cultured cells. This method is substantially less labor-intensive than manual classification and therefore allows rapid and accurate processing of images from large numbers of cells. In examining ruffle formation under several experimental conditions, we have found that this protocol provides a substantial (at least 10-fold) reduction in the time required to quantify data compared with manual methods. Given the speed with which data can be acquired using this protocol, it is well suited to the analysis of large amounts of image data obtained in time-courses during live cell experimentation. The ability to quantify ruffle formation over the entire dorsal surface of a cell makes this method suitable to the rapid and accurate assessment of the involvement of numerous cellular factors in ruffle formation. In this way, the method complements existing approaches that allow analysis of ruffling dynamics with high spatial and temporal resolution (22). Our method is also highly adaptable; the processing and classification techniques can be modified, for example by adjusting the $\sigma$ values in the 2-D DoG filter to detect lines or structures with different size characteristics, by using other line detection methods, or by demarcating cellular boundaries along more directions (e.g., x, y, and diagonally). Overall, this method is a reliable, yet rapid, approach for the automated quantification of fluorescently stained ruffles and has the potential to be applied to the analysis of other fine cellular structures.

\section{ACKNOWLEDGMENTS}

We would like to thank Dr. Leonid Brown (University of Guelph) for critical reading of the manuscript. This work was supported by the Natural Sciences and Engineering Research Council of Canada (NSERC). M.G.C. holds a Canadian Institutes of Health Research (CIHR) New Investigator Award.

\section{COMPETING INTERESTS STATEMENT}

The authors declare no competing interests.

\section{REFERENCES}

1. Abercrombie, M., J.E. Heaysman, and S.M. Pegrum. 1970. The locomotion of fibroblasts in culture. I. Movements of the leading edge. Exp. Cell Res. 59:393-398.

2.Heath, J.P. and L.D. Peachey. 1989. Morphology of fibroblasts in collagen gels: a study using $400 \mathrm{keV}$ electron microscopy and computer graphics. Cell Motil. Cytoskeleton 14:382.

3. Ridley, A.J. 1994. Membrane ruffling and signal transduction. BioEssays 16:321-327.

4. Trinkaus, J.P. 1973. Modes of cell locomotion in vivo, p. 233-244. In R. Porter and D.W. Fitzsimmons (Eds.), Locomotion of Tissue Cells. CIBA Foundation Symposium, vol. 14 Elsevier, New York.

5. Harris, A.K. 1999. A dozen questions about how tissue cells crawl, p. 315-341. In J.M. Lackie, G. Dunn, and G.E. Jones (Eds.), 
Cell Behaviour: Control and Mechanism of Motility. Biochemical Society Symposia, vol. 65. Princeton University Press, Princeton.

6. Suetsugu, S., D. Yamazaki, S. Kurisu, and T. Takenawa. 2003. Differential roles of WAVE1 and WAVE2 in dorsal and peripheral ruffle formation for fibroblast cell migration. Dev. Cell 5:595-609.

7.Saadoun, S., M.C. Papadopoulos, M. Hara-Chikuma, and A.S. Verkman. 2005. Impairment of angiogenesis and cell migration by targeted aquaporin-1 gene disruption. Nature 434:786-792.

8. Kiosses, W.B., S.J. Shattil, N. Pampori, and M.A. Schwartz. 2001. Rac recruits high-affinity integrin alphavbeta3 to lamellipodia in endothelial cell migration. Nat. Cell Biol. 3:316-320.

9. Tall, E.G., I. Spector, S.N. Pentyala, I. Bitter, and M.J. Rebecchi. 2000. Dynamics of phosphatidylinositol 4,5-bisphosphate in actin-rich structures. Curr. Biol. 10:743-746.

10. Bailly, M., J. Wyckoff, B. Bouzahzah, R. Hammerman, V. Sylvestre, M. Cammer, R. Pestell, and J.E. Segall. 2000. Epidermal growth factor receptor distribution during chemotactic responses. Mol. Biol. Cell 11:3873-3883.

11. Borm, B., R.P. Requardt, V. Herzog, and G. Kirfel. 2005. Membrane ruffles in cell migration: indicators of inefficient lamellipodia adhesion and compartments of actin filament reorganization. Exp. Cell Res. 302:83-95.

12. Enomoto, T. and Y. Asano. 1994. Induction of membrane ruffling by growth factors in morphologically TPA-resistant Balb/c3T3 TR4 cells. Cell Struct. Funct. 19:89-96.

13. Cox, D., P. Chang, Q. Zhang, P.G. Reddy, G.M. Bokoch, and S. Greenberg. 1997. Requirements for both Rac1 and Cdc42 in membrane ruffling and phagocytosis in leukocytes. J. Exp. Med. 186:1487-1494.

14. Wells, C.M., M. Walmsley, S. Ooi, V. Tybulewicz, and A.J. Ridley. 2004. Rac1deficient macrophages exhibit defects in cell spreading and membrane ruffling but not migration. J. Cell Sci. 117:1259-1268.

15. Otsu, N. 1979. A threshold selection method from gray-level histograms. 9:62-66.

16. Marr, D. and E. Hildreth. 1980. Theory of edge detection. Proc. R. Soc. Lond. B. Biol. Sci. 207:187-217.

17.Russ, J.C. 1990. Computer-Assisted Microscopy: The Measurement and Analysis of Images. Plenum Press, New York.

18. Hosmer, D.W. and S. Lemeshow. 1989. Applied Logistic Regression. Wiley \& Sons, New York.

19. Mallot, H.A. 2000. Edge detection, p. 73. In Computational Vision: Information Processing in Perception and Visual Behavior. MIT Press, Cambridge.

20. Boukerroui, D., J.A. Noble, and M. Brady. 2004. On the choice of band-pass quadrature filters. J. Math. Imaging Vis. 21:53-80.

21. Stevens, R.G. and S.A. Beaman. 1988. The use of difference of Gaussian image filtering to assess objectively the correlation between breast vascularity and breast cancer. Phys. Med. Biol. 33:1417-1431.

22. Hinz, B., W. Alt, C. Johnen, V. Herzog, and H.W. Kaiser. 1999. Quantifying lamella dy- namics of cultured cells by SACED, a new computer-assisted motion analysis. Exp. Cell Res. 251:234-243.

23.Pitas, I. 2000. Shape description, p. 323. In Digital Image Processing Algorithms and Applications. Wiley \& Sons, New York.

24. Pourghahramani, P. and E. Forssberg. 2005. Review of applied particle shape descriptors and produced particle shapes in grinding environments. Part I: Particle shape descriptors. 26:145-166.

25. Russell, K.A., S.D. Waldman, and J.M. Lee. 2000. Video-imaging assessment of nasal morphology in individuals with complete unilateral cleft lip and palate. Cleft Palate Craniofac. J. 37:542-550.

26. Rasband, W.S. 1997. ImageJ, Vol. 2003-2005 UR rsb.info.nih.gov.cerberus.lib.uoguelph.ca/ ij/docs/menus/analyze.html. NIH, Bethesda, MD.

Received 4 November 2005; accepted 21 February 2006.

Address correspondence to Marc $G$. Coppolino, Department of Molecular and Cellular Biology, University of Guelph, Guelph, ON, N1G 2W1, Canada. e-mail: mcoppoli@uoguelph.ca

To purchase reprints

of this article, contact

Reprints@BioTechniques.com 